\title{
A Randomised Comparison of Values and Goals, Versus Goals Only and Control, for High Nonclinical Paranoia
}

\author{
M. Davies ${ }^{1} \cdot$ L. Ellett ${ }^{1} \cdot$ J. Kingston ${ }^{1}$
}

Accepted: 17 April 2021 / Published online: 10 May 2021

(C) The Author(s) 2021

\begin{abstract}
Background Paranoia is common in the general population. Focusing on values and enhancing value-based acts may attenuate it. This study compared three brief (30-min, self-directed) online conditions: focusing on values and value-based goal setting $(n=30)$, goal setting only $(n=32)$ and non-values/goals control $(n=32)$ in a high paranoia sample.

Methods Participants were randomly assigned to condition. State paranoia (primary outcome) and positive and negative self-views following a difficult interpersonal experience (secondary outcome) were assessed at baseline and two-weeks.

Results Intention-to-treat: state paranoia was significantly lower following the values condition as compared to non-values/ goals control $\left(\eta \mathrm{p}^{2}=.148\right)$ and goals only $\left(\eta \mathrm{p}^{2}=.072\right)$. Only the former comparison was significant. Per-protocol: groups did not significantly differ $(p=.077)$. Within-group effect sizes: values and value-based goal setting (intention-to-treat $d=.82$, per-protocol $d=.78$ ), goals only (intention-to-treat $d=.41$, per-protocol $d=.42$ ) non-values/goals control (intention-to-treat $d=.25$, per-protocol $d=.24$ ). Positive self-views increased in all conditions. The increase was largest for the values condition, but not significantly so.

Limitations Reliance on self-report, brief follow-up, predominantly White female sample.

Conclusions The values condition was most effective at reducing non-clinical paranoia. The values condition appeared to increase positive self-views more so than comparison groups, but the sample was small and the difference was non-significant.
\end{abstract}

Keywords Values $\cdot$ Goals $\cdot$ Paranoia $\cdot$ Self-esteem

\section{Introduction}

Paranoia describes the belief that others will intentionally cause one harm (Freeman \& Garety, 2014), which can be conceptualised as existing on a continuum ranging from mild concerns of suspicion and distrust of others (e.g., others want to cause me harm), to less commonly reported delusions of persecution, which are more typical in clinical presentations (e.g., severe threat from others; Freeman et al., 2019). Paranoia is commonly reported in the general population with approximately $28 \%$ of individuals reporting elevated levels of paranoid thinking in everyday life (Freeman et al., 2019). Across the spectrum of experience, paranoia has been associated with common risk factors (e.g., urban dwelling, victimisation, childhood adversity) and

$\triangle$ J. Kingston

Jessica.kingston@rhul.ac.uk

1 Psychology Department, Royal Holloway University of London, Surrey TW20 0EX, UK psychological mechanisms (e.g., negative self-beliefs, interpretation bias, worry, negative affect; see Freeman et al., 2019). High paranoia in the general population is associated with distress, poor physical health and suicidal ideation (Freeman \& Garety, 2014). Furthermore, it can be a risk factor for future psychotic illness (e.g., Hanssen, 2005; Poulton et al., 2000; van Os et al., 2001). Relative to other psychoticlike experiences, paranoia is also more resistant to change (Allen-Crooks \& Ellett, 2014; Van der Gaag et al., 2014) and is slow to dissipate once activated (Ellett \& Chadwick, 2007). Identifying factors that attenuate paranoia is important in its own right, as well as having the potential to inform effective clinical interventions and offset the escalation of difficulties in the general population (Elahi et al., 2017).

Intervention research is increasingly interested in developing therapeutic approaches that are recovery focused; that is, "personalised, meaningful, and engaging" (Freeman, 2019) and that help people to become themselves more fully (Chadwick, 2019). One such approach, which is central to Acceptance and Commitment Therapy (ACT; 
Hayes, Strosahl \& Wilson, 2004), is helping individuals to identify and reflect on the areas in life they most care about - their values - and establishing patterns of behaviour that increase the concordance between their values and day-today actions. A few studies have investigated the independent effects of values and committed action on aspects of psychological health. For example, Villatte et al. (2016) examined the effects of an 8-week, face-to-face, values and committed action intervention on psychological distress in an adult sample who met clinical case status using the Brief Symptom Inventory (Derogatis, 1993). They reported significant improvements in distress and quality of life following the values intervention with effects maintained at 3 -month follow-up. Similar effects have been reported in student samples (e.g., Levin et al., 2020; Petersen et al., 2019). Within social psychology, the psychological benefits of brief value reflection tasks (i.e., without the addition of value-based goal setting) have been extensively investigated. Here, research demonstrates that focusing on a strongly held value or personal strength mitigates the impact of difficult experiences on the self, by helping to remind people of their broader identity and global self-worth (e.g., Sherman \& Cohen, 2006).

$\mathrm{ACT}$ as a full treatment package has shown promising effects for individuals experiencing high levels of paranoia (e.g., Shawyer et al., 2017; Tyrberg et al., 2017), but the independent effects of values and committed action for people with high levels of paranoia has not previously been investigated. Examining the impact of a values reflection task on paranoia in a predominantly student sample, Kingston and Ellett (2014) reported that participants who reflected on a core value experienced less paranoia following a threat induction than those in a non-values control group. Extending this, Evans et al. (2019) reported that the combination of values reflection plus value-based goal setting on paranoia exceeded the effects of values reflection alone when effects were examined over a two-week period. Moreover, the values plus value-based goals intervention was most effective for those with low self-esteem. This is especially relevant to individuals experiencing paranoia, as negative self-processes such as low trait self-esteem (high negative and low positive self-beliefs), unstable state selfesteem (highly fluctuating positive and negative self-beliefs) and negative core beliefs (negative global self-beliefs) have been established as causal mechanisms across the spectrum of experience (e.g., Jongeneel et al., 2018; Kesting \& Lincoln, 2013; Thewissen et al., 2008, 2011).

Overall, therefore, research suggests that reflecting on a core value and setting value-based goals may help to attenuate non-clinical paranoia, but this has only been tested in samples with mild-moderate levels. Testing the possible benefits for individuals with high non-clinical paranoia is important, not only because of the negative effects of paranoia on day to day life and well-being, but also because this research has the potential to inform our understanding and treatment of clinical paranoia. The main aim of the current study was to examine whether a brief, online values-based intervention (value identification, reflection, and setting value-based goals) could be effective in reducing paranoia (primary outcome) for individuals with high nonclinical paranoia. Whilst previous research has shown that reflecting on a core value and setting value-based goals is more effective than the reflection task alone, without a goals only comparison, it was not possible to ascertain whether the effects were driven by the combining of values and goals, or by goal setting in and of itself. In the current study, two comparisons were therefore utilised; a goal setting only condition and a non-value and goals control. Finally, we examined whether participating in the values-based intervention impacted future experiences that paranoia-prone individuals find challenging - that is, difficult interpersonal experiences. Here, we tested whether positive and negative self-views (secondary outcome), following a recent difficult interpersonal event, were higher/lower following the valuesbased intervention as compared to goals only and control. We examined positive and negative self-views independently from one another based on research showing that individuals can simultaneously hold positive and negative beliefs about the self, that these dimensions independently contribute to self-esteem and paranoia (Kestling \& Lincoln, 2013) and that they independently predict behaviour (Barrowclough et al., 2003).

\section{Method}

\section{Design}

A three-armed single-blind randomised control trial was conducted fully online and hosted at a British University. Participants completed baseline assessments (T1) before being randomised to one of three brief, self-administered, online conditions: (i) two-week value clarification, reflection and value-based goal setting task $(\mathrm{V}+\mathrm{G})$, (ii) two-week goal setting only intervention (GO) or (iii) non-values/goals control (NVGC). After the two-week period, participants recompleted baseline questionnaires (T2). This included participants being asked to identify and describe a challenging interpersonal experience occurring within the last week. Participants then rated how they felt about themselves in that interpersonal context using a series of positive and negative self-descriptors. The study protocol was approved by the host University Ethics Committee, and all participants gave online informed consent prior to participation. 


\section{Participants}

High non-clinical paranoia has previously been established using a cut-off score of 1SD above the non-clinical norm of the Paranoia Scale (Fenigstein \& Vanable, 1992; e.g., PS $>=53$, Combs \& Penn, 2004). Based on this, we recruited participants who were 18 years or older and had a PS score of 53 or greater. Power analysis using G*POWER indicated that a total sample of $n=92$ ( 31 per condition) would detect a medium-large effect size on change in state paranoia with $80 \%$ statistical power and $\alpha=0.05$. A mixed community-university sample of $n=93$ ( $82 \%$ female, $85 \%$ students, $68 \%$ White British, 12\% Asian, 10\% Other, 7\% mixed race, $4 \%$ Black) participants were recruited to the study. Participants were recruited via posts on social media, participant recruitment websites, and an online university platform for research. The sample age ranged from 18 to 47 years old $(M=21.0$ years, $S D=4.9$ years). A Consort diagram of participant flow through the study is outlined in Fig. 1.

\section{Measures}

\section{Paranoia Inclusion Criteria}

Paranoia Scale (Fenigstein \& Vanable, 1992) is a 20-item questionnaire designed to measure non-clinical paranoid thoughts (e.g., "Someone has it in for me"; "I sometimes feel as if I am being followed"). Items are rated from $1=n o t$ at all applicable to me to $5=$ extremely applicable to me and total scores range from 20 to 100 , with higher scores indicating higher levels of paranoia. The PS is a well-recognised method for detecting paranoid thoughts (Freeman, 2008), with good internal reliability $(\mathrm{a}=0.84)$, construct validity, and test-retest reliability $(\mathrm{a}=0.70)$ over 6 -months period (Fenigstein \& Vanable, 1992). Cronbach's alpha in this sample was 0.91 .

\section{Primary Outcome}

Paranoia and Depression Scale (PDS) - Paranoia subscale (Bodner \& Mikulincer, 1998) is a 7-item measure of state paranoia, developed for use in non-clinical experimental contexts. Items rate the frequency of paranoid thoughts (e.g., "I do not trust other people's intentions", "I feel like people are hostile to $m e$ "), ranging from $1=$ not at all to $7=$ very often. Total scores range from 7 to 42 . The scale has shown good construct validity when correlated with other measures of paranoia (e.g., SCL-90 paranoia subscale $(r=0.67$, $\mathrm{p}<0.001$; Bodner \& Mikulincer, 1998), and the Paranoia Scale $(r=0.53, p<0.001$; Kingston \& Ellett, 2014). Good discriminate validity has been reported when differentiating paranoia and depression in clinical groups (Bodner \& Mikulincer, 1998). In the present sample, the PDS-Paranoia subscale had good interval validity (alpha $=0.84$ ).
Fig. 1 Consort diagram outlining participant flow through the study. $\mathrm{V}+\mathrm{G}=$ values and valuebased goal setting; $\mathrm{GO}=$ goals only; NVGC = non-values/ goals control; ITT = Intention to Treat; $\mathrm{PP}=$ Per Protocol; $\mathrm{T} 1=$ baseline, $\mathrm{T} 2=2$-weeks follow-up
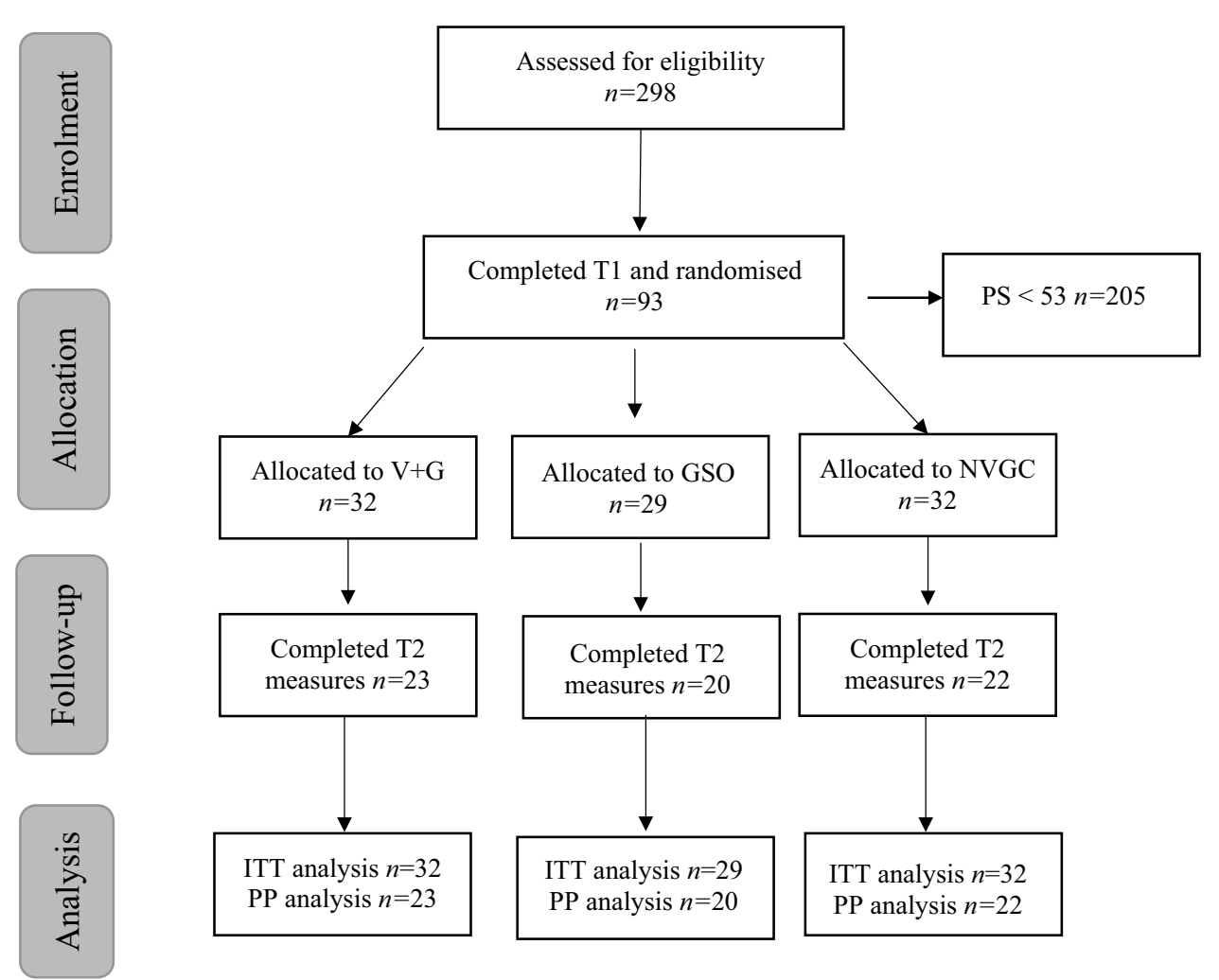


\section{Secondary Outcome}

Reactions following a difficult interpersonal event. At T1 and T2, participants were asked to "bring to mind an event that occurred in the last week involving an interaction with another person or group that you found difficult". They were asked to describe the event and why they found it difficult, as well as providing information on the number and type of people present. Following this, they were asked to rate their reactions to this event ("During the interaction I felt....") using 8 randomly ordered items from the Southampton State Self-Esteem Scale (Sedikides, 2008). These items were selected due to their interpersonal nature - i.e., they captured positive and negative self-views in an interpersonal context (negative: inadequate, stupid, unappreciated, rejected and positive: accepted, competent, effective, respected) using a 9 -point Likert scale ( $1=$ not at all to $9=$ extremely).

Goal Adherence. Self-reported goal adherence was assessed at follow-up in both active conditions through the use of a single binary item "Did you complete one of your set goals in the past two weeks?" (Response: "yes" or "no").

\section{Conditions}

\section{Values Clarification, Reflection and Value-Based Goal Setting Condition (V+G)}

A computerised version of the $\mathrm{V}+\mathrm{G}$ intervention was utilised. Participants read a brief description of values (e.g. Chase et al., 2013; Harris, 2008, 2011, 2013), after which they completed a digital card-sort task (Harris, 2008, 2013), allocating 58 cards to one of three piles: very important to me; quite important to me; not important to me (Ciarrochie $\&$ Bailey, 2008; Harris, 2008, 2013). Participants then chose a very important value to write about for ten minutes, focusing on why it is meaningful to them and describing a time it made them feel good about themselves. As a manipulation check, participants then wrote two reasons why their chosen value was important to them (Sherman et al., 2006). After this, participants completed a goal-setting task (Evans et al., 2019). Participants read a rationale for values-based goal setting and were given guidance on setting a personally meaningful values-based goal to work on over the next week using 'SMART' principles: specific, meaningful, adaptive, realistic and time-framed (Harris, 2008, 2013). All data (i.e., the card sort, the written values reflection task, the questionnaire data, and the chosen goal) was completed online and submitted to the experimenter via the online system. Two weeks later, as part of $\mathrm{T} 2$ data collection, participants were asked whether they had successfully completed their goal ("yes/no").

\section{Goal Setting Only Condition (G0)}

This condition was the same as the values-and-goals condition, but excluded the values component. That is, participants were orientated to the notion of goals and goal setting. They were then asked to generate a 'SMART' goal to complete over the next 2-week period. These instructions were identical to the values-and-goals condition. Two weeks later, participants were asked whether they had successfully completed their goal. In this condition, it was possible that the goals chosen by participants were value-based goals, despite having not been explicitly asked to do this. If so, this could invalidate the goals only condition as a comparison group. To address this, participants in this condition completed the online card sort task (described above) at the end of the study and were asked to indicate the extent to which the goal that they had chosen 2-weeks previously (i.e., as part of the goals only condition) was in line with their top value (“0”- not at all to " 10 " completely).

\section{Non-Values/Goals Control (NVGC)}

A computerised version of the non-values control condition descried by Evans et al. (2019) was utilised. This condition was similar to the initial stages of the values-and-goals condition, in that participants were informed about values and asked to sort 58 values into the above described piles. After the card sort, participants were instructed to select their least important value and to write about it from the perspective of someone else. This control condition is typically used within social psychology literature as it controls for non-specific aspects of the values task (i.e., ranking values followed by a focused writing task), but crucially does not involve reflecting on one's top values (McQueen \& Klein, 2006).

\section{Procedure}

Consenting participants completed the experiment online using Qualtrics software (Qualtrics, Provo, UT). After reading a participant information sheet and providing online consent, participants provided demographic data, and completed baseline assessments. All participants were invited to participate, but only the data of those meeting criteria for high non-clinical paranoia (described above) were utilised for the current study. After completing baseline assessments, participants were automatically randomised by Qualtrics software to one of the three experimental conditions described above. All conditions and all questionnaires were fully computerised and completed without support (i.e., fully self-help). Two-weeks later, a follow-up email with a link to post-intervention questionnaires was sent automatically to participants. The follow-up survey (T2) required 
participants to complete measures of state paranoia (PDS) and goal adherence $(\mathrm{V}+\mathrm{G}$ and $\mathrm{GO}$ only). After completing $\mathrm{T} 2$ measures, those in the GO condition completed the card sort task (see above).

\section{Analysis Strategy}

Sociodemographics and study variables for the three conditions at baseline were explored using two-sided independent $t$-tests and Pearson $\chi^{2}$-tests. Similarly, dropout, defined as those participants not completing the post-treatment assessment, were compared to those completing it, to assess systematic attrition. Two manipulation checks were conducted prior to hypothesis testing. Firstly, to examine whether participants in the $\mathrm{V}+\mathrm{G}$ condition had written about values that were important and meaningful to them, the experimenter read the essays and checked post-essay items (see methods section). Secondly, we wanted to assess whether GO participants had selected valued-goals, despite not being explicitly asked to (which could invalidate this condition as a comparison condition). We therefore examined scores on the likert scale administered at the end of the experiment, which measured the extent to which the goal that they had devised 2-weeks previously was in line with their top value.

Next, to examine the effect of conditions on state paranoia, the primary analysis was carried out as an Intention to Treat analysis (including all those randomised), including the data of all those meeting inclusion criteria. Missing data was managed using Multiple Imputation (MI) and Predictive Mean Matching in SPSS, with 10 iterations (computed per condition) calculated and averaged. Multiple imputations were conducted on each condition separately in order to limit the impact on any potential interactions between groups (Sullivan et al., 2018). Secondary analysis was computed in the "per protocol" sample, defined as those participants who 1) met inclusion criteria, 2) completed all stages of assessments and 3) reported completing all aspects of the intervention (e.g., essay provided for $\mathrm{V}+\mathrm{G}$ and NVGC, goals set for $\mathrm{V}+\mathrm{G}$ and $\mathrm{GO}$ ). The aim of the latter analysis was to examine the effects of the interventions when successfully administered to the target population.

The primary and secondary outcomes were tested using $3(\mathrm{~V}+\mathrm{G}, \mathrm{GO}, \mathrm{NVGC}) \mathrm{X} 2(\mathrm{~T} 1, \mathrm{~T} 2)$ repeated measures ANOVAs. Significant interactions were decomposed using between-group and within-subject analyses. First, oneway ANCOVAs were computed to compare $\mathrm{V}+\mathrm{G}$, GO and NVGC whilst controlling for baseline paranoia. Next, post-hoc paired samples t-tests were computed to assess the significance of change per condition. Effect sizes are also reported. For post-hoc analyses, alpha was set to $p<0.01$ to adjust for multiple comparisons.

\section{Results}

\section{Descriptives and Preliminary Analyses}

Trait paranoia scores ranged from 53-83 $(M=61.38$, median $=59, S D=8.21$ ). All variables were normally distributed and conditions were equivalent on all sociodemographic (gender: $\chi_{(2)}^{2}=0.268, p=0.874$, ethnicity: $\chi$ ${ }_{(8)}^{2}=13.07, p=0.109$; age: $\left.F_{(1,92)}=0.1 .133, p=0.327\right)$ and baseline variables (Paranoia Scale: $F_{(2,92)}=0.272, p=0.763$, state paranoia: $\left.F_{(2,92)}=0.179, p=0.837\right)$.

Essays and manipulation check items from the $\mathrm{V}+\mathrm{G}$ group were read to ensure that all participants had identified values that were highly meaningful to them. The most commonly identified values in the $\mathrm{V}+\mathrm{G}$ group were 'Authenticity' ( $\mathrm{n}=3$, example goal: "To do more things that benefit myself and that I enjoy. It can be measured by the amount of times I make a decision based on my own wants and needs") and 'Kindness to Self and Others' ( $\mathrm{n}=3$, example goal: "Ring someone that is important to me every day for at least five minutes to maintain our relationship and let them know I am thinking of them e.g. my grandparents, my sister, my auntie, friends etc".). Two people identified 'Self-development', two people identified 'Trust', and one person identified 'Acceptance', 'Contribution', 'Freedom', 'Fun', 'Gratitude', 'Humour', 'Open Mindedness', 'Respect', 'Self-Care', 'Self-Control', and 'Skilfulness'. In the NVGC group, $\mathrm{n}=8$ identified 'Power', $\mathrm{n}=3$ identified 'Romance', $\mathrm{n}=3$ identified 'Sexuality', $\mathrm{n}=2$ identified 'Conformity', $\mathrm{n}=2$ identified 'Contribution', and $\mathrm{n}=1$ identified 'Assertiveness', 'Creativity', 'Flexibility', 'Honesty', 'Independence', 'Patience', 'Self-care', and 'Sensuality'.

The final check before analysis was to assess whether GO participants had selected value-based goals, despite not being explicitly asked to. To assess this, we examined scores on the single Likert scale, which asked the extent to which the chosen goal was in line with values from their "most important to me" pile from the card sort. The mean value consistency score was low (Mean $=3.27, S D=3.78)$ suggesting that goals in this condition were not value congruent.

\section{Main Analyses: State Paranoia}

Intention-to-treat Analyses are reported in Table 1. The Time*Condition interaction was significant. Controlling for state paranoia at T1, T2 levels were significantly lower in $\mathrm{V}+\mathrm{G}$ as compared to $\operatorname{NVGC}\left(F_{(1,61)}=10.60, p=0.002\right.$, $\left.\eta \mathrm{p}^{2}=0.148\right)$. $\mathrm{T} 2$ paranoia was lower in $\mathrm{V}+\mathrm{G}$ as compared to GO, but this was not significant at the adjusted p-level of $0.01\left(F_{(1,58)}=4.52, p=0.038, \eta \mathrm{p}^{2}=0.072\right)$. GO and NVGC did not significantly differ $\left(F_{(1,58)}=1.08, p=0.303\right.$, $\left.\eta \mathrm{p}^{2}=0.018\right)$. Paired samples t-tests showed a significant 
Table 1 Means (SD), interactions, and group comparison statistics for state paranoia scores

\begin{tabular}{|c|c|c|c|}
\hline & $\mathrm{V}+\mathrm{G}$ & GO & NVGC \\
\hline \multicolumn{4}{|l|}{ ITT $(n=93)$} \\
\hline State Paranoia T1 & $24.28(7.33)$ & $23.48(8.28)$ & $23.28(5.37)$ \\
\hline State Paranoia T2 & $18.39(6.35)$ & $21.01(7.37)$ & $22.18(6.44)$ \\
\hline \multicolumn{4}{|c|}{ Condition ${ }^{*}$ Time Inter: $\mathrm{F}_{(2,90)}=5.387, p=.006$, partial $\eta^{2}=.107$} \\
\hline \multicolumn{4}{|l|}{$\mathrm{PP}(\mathrm{n}=65)$} \\
\hline State Paranoia T1 & $24.78(6.63)$ & $23.00(7.39)$ & $23.00(5.29)$ \\
\hline State Paranoia T2 & $18.91(7.04)$ & $20.45(7.98)$ & $21.82(7.18)$ \\
\hline \multicolumn{4}{|c|}{ Condition ${ }^{*}$ Time Inter: $\mathrm{F}_{(2,62)}=3.27, p=.045$, partial $\eta^{2}=0.095$} \\
\hline
\end{tabular}

$\mathrm{V}+\mathrm{G}=$ values and value-based goal setting; $\mathrm{GO}=$ goals only; $\mathrm{NVGC}=$ non-values/goals control

reduction in paranoia from $\mathrm{T} 1-\mathrm{T} 2$ for $\mathrm{V}+\mathrm{G}\left(t_{(31)}=4.63\right.$, $p<0.001, d=0.82)$ and GO $\left(t_{(31)}=2.206, p=0.036\right.$, $d=0.41)$, but not NVGC $\left(t_{(31)}=1.40, p=0.172, d=0.25\right)$.

Per Protocol Analysis Also showed a significant Time*Condition interaction. Controlling for T1 state paranoia, $\mathrm{T} 2$ scores were lower in $\mathrm{V}+\mathrm{G}$ as compared to NVGC and GO, but not significantly so with p-values adjusted (NVGC: $F_{(1,45)}=4.79, p=0.034, \eta \mathrm{p}^{2}=0.102$ and GO: $\left.F_{(1,43)}=1.74, p=0.195, \eta \mathrm{p}^{2}=0.042\right)$. Paired samples t-tests showed a significant reduction in paranoia from T1-T2 for $\mathrm{V}+\mathrm{G}\left(t_{(22)}=3.74, p=0.001, d=0.78\right)$, but not for $\mathrm{GO}$ $\left(t_{(19)}=1.87, p=0.077, d=0.42\right)$, or NVGC $\left(t_{(21)}=1.11\right.$, $p=0.281, d=0.24)$.

\section{Goal Completion}

In both the $\mathrm{V}+\mathrm{G}$ and $\mathrm{GO}$ condition, $61 \%$ of participants reported completing their goals.

\section{Reactions to a Difficult Interpersonal Event}

Due to an administration error, 12 participants did not receive the reactions to interpersonal events measure. A further nine received it but did not complete it. Thus, analyses were based on $n=44(V+G n=14$, GO $n=16$ and NVGC $n=14$ ). Figure 2 illustrates mean $T 1$ and $T 2$ scores for positive and negative self-views per group. Inspection of the means suggested that baseline negative self-views were lower in the $\mathrm{V}+\mathrm{G}$ than $\mathrm{GO}$ and $\mathrm{NGVC}$, but not significantly so $\left(F_{(2,43)}=2.24, p=0.12\right)$. For all groups, negative selfviews appeared to be relatively stable from $\mathrm{T} 1-\mathrm{T} 2$, suggesting conditions did not affect negative self-views. For positive self-views, means suggested equivalence across groups at baseline and improvements in positive self-views from T1-T2 in all conditions. $V+G$ showed the greatest increase. A mixed repeated measures ANOVA indicated a main effect
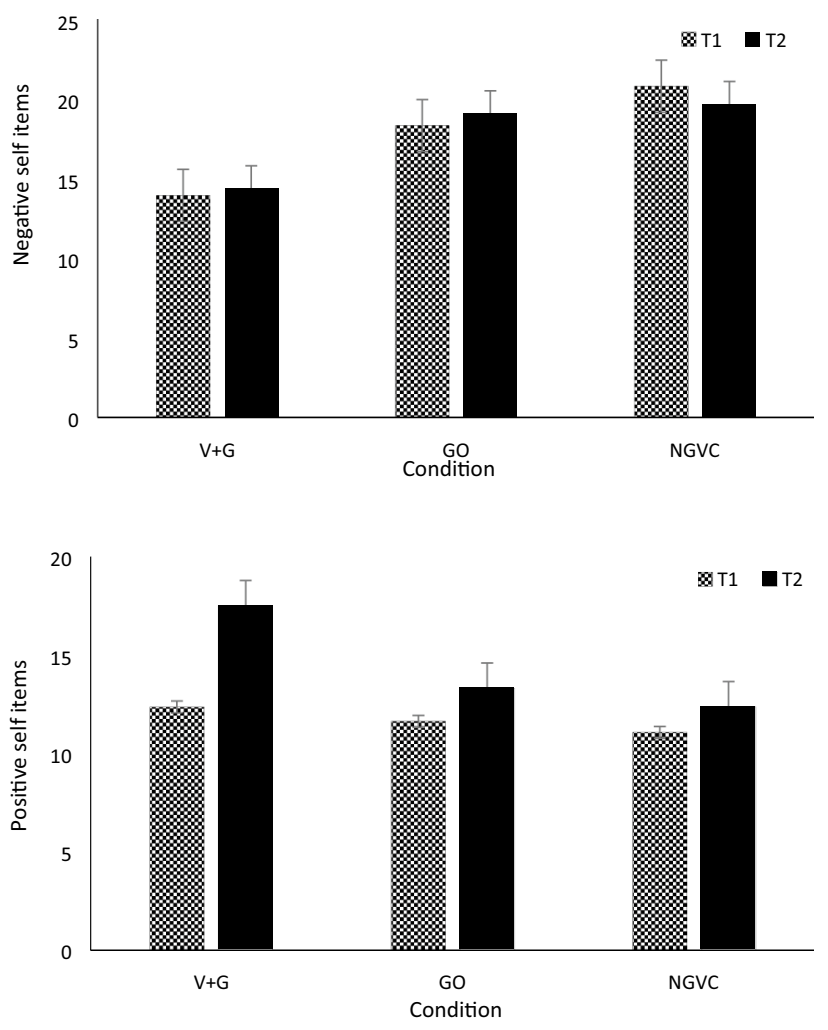

Fig. 2 Endorsement of positive and negative self-evaluations following a difficult interpersonal event at T1 (baseline) and T2 (2-week follow-up) with standard error bars. Higher scores reflect higher negative self-views (top) and higher positive self-view (bottom). $\mathrm{V}+\mathrm{G}=$ values and value-based goal setting; $\mathrm{GO}=$ goals only; $\mathrm{NVGC}=$ non-values/goals control

of Time $\left(F_{(1,41)}=5.377, p=0.025\right)$ but the Time*Condition interaction was not significant.

\section{Discussion}

The current findings suggest that individuals with high levels of non-clinical paranoia engaged with these brief online and self-help tasks, with levels of drop-out (30\%) comparable to online interventions for other presentations (see Melville et al., 2010). Results from the ITT analysis indicated that a values clarification and reflection task followed by valuesbased goal setting (i.e., $\mathrm{V}+\mathrm{G}$ condition) led to lower levels of state paranoia after a 2-week period as compared to goal setting alone and a non-values/goals control. Paranoia scores were significantly lower for $\mathrm{V}+\mathrm{G}$ as compared to the no values/goals control (large ES) whereas scores were lower, but not significantly so, when comparing $\mathrm{V}+\mathrm{G}$ to goals only (medium ES). Significant within-subject's reductions in paranoia were reported for $\mathrm{V}+\mathrm{G}$ and $\mathrm{GO}$, with uncontrolled effect sizes of $d=0.82$ and $d=0.41$ respectively. In the per protocol sample there were no significant between-group 
effects over time. Looking at within-group change, the effect sizes were comparable to the ITT analysis, but only the $\mathrm{V}+\mathrm{G}$ condition showed significant reductions in paranoia. Contrasting ITT and PP findings, the between group differences were greater in the ITT sample. This may have occurred because the $\mathrm{V}+\mathrm{G}$ condition retained individuals with higher baseline state paranoia (i.e., the baseline mean for the ITT sample was lower than that of the PP sample), whereas the reverse occurred for GO and NVGC. Overall, these findings suggest that focusing on a core value and pursuing value-based action can affect a reduction in paranoia to a greater extent than setting and pursuing goals that are not formulated within the context of an individual's values. Tentatively, this finding suggests that embedding goal setting-a common and well utilised therapeutic technique-within an individual's core values enhanced the effectiveness of the intervention.

The current study also sought to investigate whether conditions differentially impacted positive and negative self-views following a difficult interpersonal experience. Although the three conditions did not significantly differ on positive or negative self-views over the two-week period, mean scores suggested two noteworthy observations: (a) none of the conditions effected negative self-views overtime and (b) positive self-views increased in all conditions over time, but noticeably more so for those in the $\mathrm{V}+\mathrm{G}$ condition. These findings must be interpreted with caution, given the small sample and non-significant findings. Tentatively, they suggest that $\mathrm{V}+\mathrm{G}$ participants evaluated themselves more positively following a difficult interpersonal event than participants in the other two conditions. Research has not previously investigated the impact of values work on selfviews following difficult experiences; however, these findings complement those of Jaremka et al. (2011) who found that reflecting on an important aspect of the self reduced subsequent distancing from others following a lab-based interpersonal threat. Future research would benefit from testing the replicability of this finding in a larger sample, in standardised settings and in real time, such as by using virtual reality or a social interaction role play. If replicated, it would be useful to examine the specifics of this effect, such as whether enhanced positive self-views mediates the effect of $\mathrm{V}+\mathrm{G}$ on paranoia.

The current findings should be considered in light of several limitations. Firstly, the impact of the conditions was not examined over the longer term and, as such, it is not possible to ascertain whether the observed effects are durable. Secondly, there are measurement issues for both the primary and secondary outcome. Both relied on self-report, and the secondary outcome measure-reporting on past negative events-is likely to have involved different types of recall bias. Furthermore, the effect of concurrent difficulties such as, for example, depression and anxiety were not controlled for. Future research would benefit from including a range of indices of paranoia, such as by examining the impact of the interventions on behavioural outcomes in a virtual reality environment or an artificially constructed, standardised, difficult interpersonal event (e.g., Cyberball, social interaction role play). Thirdly, whilst all participants presented with high levels of paranoia, the sample was nonetheless predominantly white and female. As such, several of the characteristics known to be associated with high levels of paranoia (e.g., ethnic minority groups, males) were not represented. This limits the generalisability of the findings. Finally, paranoia is a multifaceted experience and future research would benefit from examining belief dimensions such as distress, conviction and impact on functioning.

Notwithstanding these limitations, the study has some tentative implications. Firstly, the findings suggest that a brief values clarification, reflection and values-based goals intervention may provide a low intensity and non-pathologising method for attenuating high levels of paranoia in non-clinical groups. Secondly, goal setting is a commonly utilised and transdiagnostic clinical method that is a cornerstone of many cognitive behavioural interventions. The current data tentatively suggest that the effects of goal setting on paranoia were enhanced by rooting this process in an individual's values system. Future research would benefit from examining whether the differences observed here are evident for other areas of distress, with the potential for enhancing goals-based interventions.

Author Contributions All authors contributed to study conception and design. Material preparation and data collection were performed by $\mathbf{M}$. Davies, analysis by M. Davies and J Kingston. The first draft of the manuscript was written by J. Kingston and all authors read, commented on, and approved the final draft.

Funding No funding.

Data Availability The datasets generated during and/or analysed during the current study are available from the corresponding author on reasonable request.

\section{Declarations}

Conflict of Interest The authors have no relevant financial or non-financial interests to disclose. The authors have no conflicts of interest to declare that are relevant to the content of this article. All authors certify that they have no affiliations with or involvement in any organization or entity with any financial interest or non-financial interest in the subject matter or materials discussed in this manuscript.

Ethical Approval Approval was obtained from the Royal Holloway Research Ethics Committee (ID: 180). The procedures used in this study adhere to the tenets of the Declaration of Helsinki.

Informed Consent Informed consent was obtained from all individual participants included in the study. 
Research Involving Human and Animal Participants No animal studies were carried out by the authors for this article.

Open Access This article is licensed under a Creative Commons Attribution 4.0 International License, which permits use, sharing, adaptation, distribution and reproduction in any medium or format, as long as you give appropriate credit to the original author(s) and the source, provide a link to the Creative Commons licence, and indicate if changes were made. The images or other third party material in this article are included in the article's Creative Commons licence, unless indicated otherwise in a credit line to the material. If material is not included in the article's Creative Commons licence and your intended use is not permitted by statutory regulation or exceeds the permitted use, you will need to obtain permission directly from the copyright holder. To view a copy of this licence, visit http://creativecommons.org/licenses/by/4.0/.

\section{References}

Allen-Crooks, R., \& Ellett, L. (2014). Naturalistic change in nonclinical paranoid experiences. Behavior and Cognitive Psychotherapy, 42, 634-639. https://doi.org/10.1017/S1352465813001148

Barrowclough, C., Tarrier, N., Humphreys, L., Ward, J., Gregg, L., \& Andrews, A. (2003). Self-esteem in Schizophrenia: Relationships between self-evaluation, family attritudes and Symptomatology. Jouranl of Abnormal Psychology, 112, 92-99. https://doi.org/10. 1037/0021-843X.112.1.92

Bodner, E., \& Mikulincer, M. (1998). Learned helplessness and the occurrence of depressive-like and paranoid-like responses: The role of attentional focus. Journal of Personality and Social Psychology, 74, 1010-1023. https://doi.org/10.1037/0022-3514.74.4. 1010

Chadwick, P. (2019). Mindfulness for psychosis: A humanising therapeutic process. Current Opinions in Psychology, 28, 317-320. https://doi.org/10.1016/j.copsyc.2019.07.022

Chase, J. A., Houmanfar, R., Hayes, S., Ward, T. A., Plumb Vilardaga, J., \& Follette, V. (2013). Values are not just goals: Online ACTbased values training adds to goal setting in improving undergraduate college student performance. Journal of Contextual Behavioural Science, 2, 79-84. https://doi.org/10.1016/j.jcbs. 2013.08.002

Ciarrochi, J., \& Bailey, A. (2008). A CBT practitioner's guide to ACT: How to bridge the gap between cognitive behavioral therapy and acceptance and commitment therapy. Oakland, CA: New Harbinger.

Combs, D., \& Penn, D. (2004). The role of subclinical paranoia on social perception and behavior. Schizophrenia Research, 69, 93-104. https://doi.org/10.1016/S0920-9964(03)00051-3

Derogatis, L. R. (1993). BSI: Administration, scoring \& procedures manual for the Brief Symptom Inventory (3rd ed.). Minneapolis, MN: National Computer Systems.

Elahi, A., Algorta, G. P., Varese, F., McIntyre, J. C., \& Bentall, R. P. (2017). Do paranoid delusions exist on a continuum with subclinical paranoia? A multi-method taxometric study. Schizophrenia research, 190, 77-81

Ellett, L., \& Chadwick, P. (2007). Paranoid cognitions, failure, and focus of attention in college students. Cognition and Emotion, 21, 558-576. https://doi.org/10.1080/02699930600758155

Evans, N., Carpenter, R., Ellett, L., \& Kingston, J. (2019). Immediate and short term effects of values-based interventions on paranoia. Journal of Behavior Therapy and Experimental Psychiatry. https://doi.org/10.1016/j.jbtep.2019.101500
Fenigstein, A., \& Vanable, P. (1992). Persecutory ideation and selfconsciousness. Journal of Personality and Social Psychology, 62, 129-138. https://doi.org/10.1037//0022-3514.62.1.129

Freeman, D. (2008). The assessment of persecutory ideation. In D. Freeman, R. Bentall, \& P. Garety (Eds.), Persecutory delusions: Assessment, theory and treatment. (pp. 23-52). Oxford University Press.

Freeman, D., \& Garety, P. (2014). Advances in understanding and treating persecutory delusions: A review. Social Psychiatry and Psychiatric Epidemiology, 49, 1179-1189. https://doi.org/10.1007/ s00127-014-0928-7

Freeman, D., Taylor, K., Molodynski, A., \& Waite, F. (2019). Treatable clinical intervention targets for patients with Schizophrenia. Schizophrenia Research, 211, 44-50. https://doi.org/10.1016/j. schres.2019.07.016

Hanssen, M., Bak, M., Bijl, R., Vollenbergh, W., \& van Os, J. (2005). The incidence and outcome of subclinical psychotic experiences in the general population. British Journal of Clinical Psychology, 44, 2. https://doi.org/10.1348/014466505X29611

Harris, R. (2008). The happiness trap. Robinson.

Harris, R. (2011). The Confidence Gap. Little Brown Book Group.

Harris, R. (2013). Getting unstuck in ACT: A clinician's guide to overcoming common obstacles in acceptance and commitment therapy. New Harbinger.

Hayes, S. C., Strosahl, K. D., \& Wilson, K. G. (2004). Acceptance and commitment therapy: An experiential approach to behaviour change. Guilford Press.

Jaremka, L. M., Bunyan, D. P., Collins, N. L., \& Sherman, D. K. (2011). Reducing defensive distancing: Self-affirmation and risk regulation in response to relationship threats. Journal of Experimental Social Psychology, 47, 264-268. https://doi.org/ 10.1016/j.jesp.2010.08.015

Jongeneel, A., Pot-Kolder, R., Counotte, J., van de Gaag, M., \& Veling, W. (2018). Self-esteem moderates affective and psychotic responses to social stress in psychosis: A virtual reality study. Schizophrenia Research, 202, 80-85

Kesting, M., Bredenpohl, M., Klenke, J., Westermann, S., \& Lincoln, T. (2013). Journal of Behavior Therapy and Experimental Psychiatry, 44, 122-128. https://doi.org/10.1016/j.jbtep.2012. 07.010

Kesting, M.-L., \& Lincoln, T. M. (2013). The relevance of self-esteem and self-schemas to persecutory delusions: A systematic review. Comprehensive Psychiatry, 54, 766-789. https://doi.org/10. 1016/j.comppsych.2013.03.002

Kingston, J., \& Ellett, L. (2014). Self-affirmation and non-clinical paranoia. Journal of Behaviour Therapy and Experimental Psychiatry, 45, 502-505. https://doi.org/10.1016/j.jbtep.2014.07.004

Levin, M., Krafft, J., Hicks, E., Pierce, B., \& Twohig, M. (2020). A randomized dismantling trial of the open and engaged components of acceptance and commitment therapy in an online intervention for distressed college students. Behaviour Research and Therapy. https://doi.org/10.1016/j.brat.2020.103557

McQueen, A., \& Klein, M. (2006). Experimental manipulations of selfaffirmation: A systematic review. Self and Identity, 5, 289-354

Melville, K., Casey, L., \& Kavanagh, D. (2010). Drop out from internet-based treatment for psychological disorders. British Journal of Clinical psychology, 49, 445-471. https://doi.org/10.1348/01446 6509X472138

Petersen, J., Krafft, J., Twohig, M., \& Levin, M. (2019). Evaluating the open and engaged components of acceptance and commitment therapy (ACT) in an online self-guided website: Results from a pilot trial. Behavior Modification. https://doi.org/10.1177/01454 45519878668

Poulton, R., Caspi, A., Moffitt, T. E., Cannon, M., Murray, R., \& Harrington, H. (2000). Children's self-reported psychotic symptoms and adult schizophreniform disorder. Archives of General 
Psychiatry, 57, 1053-1058. https://doi.org/10.1001/archpsyc.57. 11.1053

Sedikides, C. (2008). The Southampton State Self-Esteem Scale. Unpublished manuscript, University of Southampton, England.

Shawyer, F., Farhall, J., Hayes, S. C., Gallop, R., Copolov, D., \& Castle, D. J. (2017). Acceptance and Commitment Therapy for psychosis: Randomised controlled trial. British Journal of Psychiatry, 210(2), 140-148. https://doi.org/10.1192/bjp.bp.116.182865

Sherman, D., \& Cohen, G. (2006). The psychology of self-defence: Self-affirmation theory. Advances in Experimental Social Psychology, 38, 183-242. https://doi.org/10.1016/j.schres.2018.06.042

Smout, M., Davies, M., Burns, N., \& Christie, A. (2014). Development of the Valuing Questionnaire (VQ). Journal of Contextual Behavioral Therapy. https://doi.org/10.1016/j.jcbs2014.06.001

Sullivan, T. R., White, I. R., Salter, A. B., Ryan, P., \& Lee, K. J. (2018). Should multiple imputation be the method of choice for handling missing data in randomized trials? Statistical Methods in Medical Research, 27(9), 2610-2626. https://doi.org/10.1177/09622 80216683570

Thewissen, V., Bentall, R. P., Lecomte, T., van Os, J., \& Myin-Germeys, I. (2008). Fluctuations in self-esteem and paranoia in the context of daily life. Journal of Abnormal Psychology, 117, 143153. https://doi.org/10.1037/0021-843x.117.1.143

Thewissen, V., Bentall, R., Oorschot, M., Campo, J., van Lierop, T., van Os, J., \& Myin-Germeys, I. (2011). Emotions, self-esteem, and paranoid episodes: An experience sampling study. British Journal of Clinical Psychology, 50(2), 178-195. https://doi.org/ 10.1348/014466510X508677

Tyrberg, M., Carlbring, P., \& Lundgren, T. (2017). Nordic Psychology, 69(2), 110-125. https://doi.org/10.1080/19012276.2016.1198271

van der Gaag, M., Valmaggia, L. R., \& Smit, F. (2014). The effects of individually tailored formulation-based cognitive behavioural therapy in auditory hallucinations and delusions: A meta-analysis. Schizophrenia research, 156(1), 30-37. https://doi.org/10.1016/j. schres.2014.03.016

van Os, J., Hanssen, R., Bijl, W., \& Vollenbergh, W. (2001). Prevalence of psychotic disorder and community level of psychotic symptoms: An urban-rural comparison. Archives of General Psychiatry, 58, 663-668. https://doi.org/10.1001/archpsyc.58.7.663

Villatte, J. L., Vilardaga, R., Villatte, M., Vilardaga, J. C. P., Atkins, D. C., \& Hayes, S. C. (2016). Acceptance and Commitment Therapy modules: Differential impact on treatment processes and outcomes. Behaviour research and therapy, 77, 52-61. https://doi. org/10.1016/j.brat.2015.12.001

Publisher's Note Springer Nature remains neutral with regard to jurisdictional claims in published maps and institutional affiliations. 